Rev. Chis. Pediatr. 58(1): 61-65, 1987

\title{
Esferocitosis hereditaria en el período de recién nacido
}

\author{
Dr. Lautaro Vargas P. 1 ; Dra. Susana Messen H. ${ }^{1}$; Dra. Flizabeth Hubach K. ${ }^{2}$ \\ Di. Hugo García U. 1 \\ Clinical manifestations of heredity spherocytosis \\ in the neonatal period
}

The clinical and hematological features of seven newborn infants with hereditary spherocy tosis (HS) wert analyzed, 5 of them had familial antecedents of the disease. All 7 patients with neonatal HS had early and intense jaundice. Phototherapy was employed in all of them, but 3 required exchange transfusions. The evolution of these patients showed the same patlerns that those cases whose IJS diagnosis was established at later ages. Another 29 cases, recognized after the neonatal period, were also analized. In this later patients pathological neonatal jaundice was noted in $44.8 \%$ of the cases. Probably $\mathrm{HS}$ with hemolytic jaundice in the newborns is underdiagnosed and should be investigated in every patient with neonatal jaundice of unknown origin: familial antecedents of JIS tmust. be inguired, specially it there is anemia, and complete hematological study (osmotic fragility test includod) toghether with a differential diagnosis with ABO incompatibility should also be done.

(Key words: Hereditary spherocytosis, signs and symptoms, neonatal period, newborn infants, early manifestations)

La esferocitosis hereditaria (EH) es la anemia hemolítica congénita más frecuente en Chile y tambiën en los países del norte de Europa y en la población de raza blanca de Estados Unidos, pais en el que se estima que tiene una incidencia de un caso nuevo por cada 5 a 15.000 habitantes ${ }^{1,2}$. En nuestro país no existen estadísticas de incidencia aunque nuestra impresión es que debe ser menor a las cifras norteamericanas.

Si bien la $\mathbf{E H}$ es una enfermedad congenita $y$ por lo tanto presente en el período neonatal. no es frecuente formular el diagnóstico en esta edad. Sólo hemos encontrado dos referencias de EH de presentación neonatal en los últimos diez $\operatorname{años}^{3}, 4$.

1. Servicjo de Pediatría, Hospital San Juan de Dios. Departamento de Pediatría, División Ciencias Médicas Occidente, Facultad de Medicina, Universidad de Chilt.

2. Becario. Departamento de Pediatría, División Ciencias Médicas Occidente, Facultad de Medicina, Universidad de Chile.
Hasta 1983 se habian comunicado 29 recién nacidos (RN) con $E^{3}$. En una comunicación anterjor en que se revisó la casuística de 25 casos pediátricos del hospital San Juan de Dios, en sólo $2(8 \%)$ se habia formulado el diagnóstico en el período del RN ${ }^{5}$. En los últimos años de 11 nuevos casos del mismo centro, 5 fueron diagnosticados en el período neonatal lo que parece debido a una actitud de búsqueda de la enfermedad en RN con hiperbilirrubinemia de causia no explicada $\mathrm{y}$ en los RN hermanos de pacientes diagnosticados con anterioridad.

Por las consideraciones anteriores hemos creído de interés analizar las características clínicas y hematológicas de la EH con el períado neonatal.

También se analizaron retrospectivamente, a modo de referencia, otros 29 casos de EH diagnosticados después del período neonatal en la unidad de hematología del hospital San Juan de Dios en los últimos 15 años (1969-1984).

\section{MATERIAL Y METODO}

Se cstudiaron en forma retrospectiva 7 casos de $\mathbf{E H}$ 
cuyo diazmóstico se planteố en el período del recién nacido, confirmándose en todos con un completo estudio realizado en el periodo de lactante o preescolar. El diagnóstico de EH se basó en la presencia de unemia regenerativa con características hemolíticas de curso crúnico, importante esferocitosis en el estudio del frotis sanguineo $(++a++)$ y aumento de la fragilidad osmótica.

En la mayoría de los casos se revisaron los frotis sanguíneos de ambos padres. En algunos enfermos se comprtetó el estudio con exámenes complementarios como autohemólisis, sobrevida del glóbulo rojo con $\mathrm{Cr}^{51}$, captación espléniça con $\mathrm{Cr}^{51}$. mislograma.

Se registrajorl las prinçipales características clínicas y de laboratorio que presentaron los pacientes en el periodo de recién nacido y también en la eyolución ulterior.

Los enfermos han sido seguidos por lo menos por uno de $\operatorname{los}$ autores durante periodos entre 5 mescis a 6 años. Para el seguimiento se definió como formas graves de EH las que presentan 5 o más crisis hemolíticas o aplásticas que requieren transfusiones o las que permanchtemente tienen hematocritos entre 20 y $25 \%$ Las formas lever son las que no presentan antmia o esta es discreta y en los que la alteración de la fragilidad osmótica cs leve o sólo se manifiesta después de incubación durante 24 horas. Las formas moderadas ticnen caracteristivas intermedias entre las graves $y$ las leves.

\section{RESULTADOS}

Las características clinicas y hematológicas de los pecientes se describen en la Tabla 1.

Cuatro de los 7 reciên nacidos eran mujeres $(57 \%)$. Todos los enfermos tuvieron anemia e ictericia en el período neonatal y cinco tenian antecedentes familiares de EH ( $71 \%)$. La ictericia fue intensa en los siete casos (mayor que $13.2 \mathrm{mg}$ $\mathrm{x}$ dl de bilirrubinemia total) y alcanzó su máximo en el tercer o cuarto dia, salvo un caso que a las 10 horas habia alcanzado la cifra antes descrita, debiendo ser sometido a exsanguinotransfusion. Todos los recién nacidos fueron tratados con fototerapia y en 3 fue necesario realizar recambio sanguineo.

Todos los enfermos presentaron anemia intensil que fue severa en 3 (hematocrito $<30 \%$ ) y todos recibieron transfusión de glóbulos rojos, que debió repetirse en 2 enfermos. En los 7 la anemia tenía características hemolíticas con reticulocitos entre 8 y $15,5 \%$ policromatofilia $y$ microesferocitosis moderada at intensa $(++$ a +++ ). Las pruebas de Coombs fueron siempre negativas, no se detecto incompatibilidad $\mathbf{R h} y$ sólo en un caso habia incompatibilidad de grupo clásico. Tres de los 7 nifros tenian esplenomegalia entre 1 y $2 \mathrm{~cm}$ bajo el reborde costal.

Las pruebas de fragilidad osmótica realizadas entre los 3 y 12 meses de edad estuvieron alteradas en los 6 casos en que se practicaron. El seguimiento de estos niños ha durado entre 5 meses y 6 años, en sólo 2 es menor que 1 año. La mayorja de los enfermos han tenido un curso lève o moderado con la única excepción de un caso que debió ser esplenectomizado a la edad de 5 años.

De Jos 29 casos con EH detectada después del período neonatal $18(62 \%)$ eran mujeres y 20 (69\%) tenián 3 o más años de edad en el momento del diagnóstico, y $58,6(17 / 29)$ de los cusos tenian antecedentes familiares.

En contraste con los hallazgos en el recién nacido la forma de presentación más frecuente fue la anemia aislada $37,9 \%(11 / 29)$ mientras que la forma ictérico anémica se encontró en $31,1 \%$ (ver Tabla 2).

Del total de 29 enfermos, en $13(44,8 \%)$ fueron registrados antecedentes de ictericia neonatal. que en 7 casos habían obligado a usar fototerapia y en 3 exsanguinotransfusión, sufriendo uno de estos últimos secuelas neurológicas sugerentes de kernícterus.

Tabla 1.

Caracter ísticas clínicas y hematológicas de 7 casas esferocitosis hereditaria identificada en ol período neonatal

\begin{tabular}{|c|c|c|c|c|c|c|c|}
\hline Nombres & $\mathrm{CH}$ & $\mathrm{XS}$ & $\mathbf{K M}$ & CS & GB & $\mathrm{CR}$ & $\mathrm{MN}$ \\
\hline Antecodente familiar & + & + & + & + & - & + & - \\
\hline lctericia clínica $(+\mathrm{a}+++)$ & ++ & + & +4 & ++ & + & ++ & + \\
\hline Palidez $(+a++)$ & ++ & ++ & + & ++ & +++ & ++ & + \\
\hline Fisplenomegalia $\mathrm{cm} . \mathrm{s}$ & 1 & 2 & 1 & - & - & - & - \\
\hline Hematocrito $\%$ & 34 & 31 & 28 & 27 & 24 & 33 & 33 \\
\hline Reticulocitos $(+a+++)$ & 10 & 15,5 & 10 & 10 & 8 & 13 & 13,5 \\
\hline Microesferocitos $(+a+++)$ & + & ++ & ++ & + & $+1+$ & ++ & ++ \\
\hline Bilirrubinemia mg\% & 18,6 & 16 & 21,3 & 16 & 16 & 13,2 & 13,2 \\
\hline Fototcrapia & SI & SI & S] & SI & SI & SI & S! \\
\hline Recambio & 1 & NO & 1 & NO & $\mathrm{NO}$ & $\mathrm{NO}$ & 1 \\
\hline Trnasfusiones G.R. & 1 & 1 & 2 & 1 & 3 & 1 & 1 \\
\hline Año & 1975 & 1978 & 1982 & 1982 & 1983 & 1984 & 1984 \\
\hline
\end{tabular}


Tabla 2.

Forma de prescrtación de 29 pacientes con esferocitosis hereditaria identifieados después del período neonatal.

\begin{tabular}{lrr}
\hline Anemia aislada & 11 & $37,9 \%$ \\
lotericiá y anemia & 9 & $37,1 \%$ \\
Asintomática & 4 & $13,8 \%$ \\
Ictericia aislada & 3 & $10,3 \%$ \\
Crisis aplástica & 2 & $6,9 \%$ \\
\hline \multicolumn{1}{c}{ Total } & 29 & 100 \\
\hline
\end{tabular}

\section{DISCUSION}

La EH tiene frecuentemente expresión clínica en el RN aunque en sólo la minoría de los casos se planted o confirma el diagnóstico. En la Tabla 3 se detallan las principales caracter isticas hematológicas de los casos de EH neonatal comunicados en la literatura $3.6-17$

Las manifestaciones mas frecuentes de la $\mathbf{E H}$ en el RN son ictericia y anemia hemolítica con esferocitosis en el frotis sanguineo a las que se agregan los antecedentes familiares y el aumento de la fragilidad osmót ica de los glóbulos rojos.

En nuestra casuística de E.H diagnosticada después del periodo neonatal y en otras series ${ }^{6}$ cxiste el antecedente de ictericia importante en casi la mitad de los casos.

En la mayoría de las EH disgnosticadas en e! período neonatal la ictcricia se hace evidente en las primeras 48 hrs. pero también puede tardar hasta una semana en aparecer ${ }^{2}$. En nuestro estudio fue precoz pero de ascenso lento llegando al máximo entre el tercero y cuarto día y puede alcanzar ocasionalmente magnitudes peligrosas que obligan a efectuar exsanguinotransfusión. Hay varios casos de kernicterus en la litera. tura $8,9,10,16$

Con el recambio sanguíneo la hemólisis disminuye significativamente ya que se reemplazan los esferocitos, de sobrevida muy corta, por eritrocitos normales. Este hecho explica que generalmente no se produce rebote de la ictericit como ccurrió con nuestros casos. en la literatura sólo hay un caso que necesjtó dos exsanguinotransfusiones 6 .

La otra manifestación relevante de la $\mathrm{EH}$ de presentación neonatal es la anemia que puede alcanzar importante magnitud como ocurrio en todos nuestros casos, que cursaron con menos de $35 \%$ de hematocrito o menos de $15 \mathrm{~g} \mathrm{x}$ dl de hemoglobina. En la literatura, sólo 10/23 $(43,5 \%)$ presentaron menos de $15 \mathrm{~g} \mathrm{x}$ d) de hemoglobina. Trucco observó que al coexistir la $\mathbf{E H}$ con incompatibilidad de grupo clásico la anemia es siempre muy intensa ${ }^{7}$ lo que también com. probamos en un caso. La anemia se corrige con transfusión de glóbulos rojos y/o con la exangujnotransfusión. En ocasiones es necesario repetir las transfusiones.

La presencia de esferocitosis en el frotis sanguíneo se observó en todos nuestros casos siendo moderada en la mayoría (6/7) y marcada sólo en

Tabta 3.

Haljuzgos hematológicos en esferccitosis de presentación neonatal (Revisión de li literatura) (modificado de Schröter y Kashnilz) ${ }^{3}$

\begin{tabular}{|c|c|c|c|c|c|c|c|c|c|c|}
\hline & \multirow{2}{*}{$\begin{array}{c}\text { No } \\
\text { pacjentes }\end{array}$} & \multicolumn{2}{|c|}{$\begin{array}{c}\text { Jemoglobinw* } \\
\text { g;d! }\end{array}$} & \multicolumn{2}{|c|}{$\underset{\%}{\text { Reticulocitos** }}$} & \multicolumn{2}{|c|}{$\begin{array}{c}\text { Biliruvbina** } \\
\text { mg/dl }\end{array}$} & \multirow[b]{2}{*}{$>20$} & \multirow[t]{2}{*}{ Esterocit. } & \multirow[t]{2}{*}{ Recamb } \\
\hline & & $\leqslant 15$ & $>15$ & $\leqslant 10$ & $>10$ & $\leqslant 0$ & 10 a 20 & & & \\
\hline Schroter ${ }^{3}$ & 5 & 2 & 3 & 3 & 2 & 1 & 3 & 1 & 3 & 1 \\
\hline Agarwal ${ }^{4}$ & 1 & & 1 & & J & & 1 & & 1 & \\
\hline Stamcy ${ }^{6}$ & 4 & 3 & 1 & 1 & 2 & & & 4 & 4 & 4 \\
\hline Truceso ${ }^{7}$ & 7 & 5 & 2 & 6 & & ] & 5 & 1 & 6 & 4 \\
\hline Roddy ${ }^{10}$ & 2 & & 2 & & & & & 1 & & 1 \\
\hline Slapiro ${ }^{12}$ & 1 & & 1 & & & & & & 1 & \\
\hline Betke 14 & 1 & & 1 & 1 & & & & 1 & 1 & 1 \\
\hline Mazaulay ${ }^{15}$ & 1 & & 1 & & & & & & & \\
\hline$O l \operatorname{sen}^{16}$ & 1 & & & & 1 & & & 1 & 1 & 1 \\
\hline Robinson 17 & 1 & & 1 & & & & 1 & & & 1 \\
\hline Kostinas $^{23}$ & 3 & & & 1 & & & $I$ & & 2 & 1 \\
\hline Lejouge & 2 & & & 1 & 1 & & 1 & 1 & & \\
\hline Nuestro extudio & 7 & 7 & & 1 & 6 & & 6 & 1 & 7 & 3 \\
\hline Total & 36 & 17 & 13 & 14 & 13 & 2 & 17 & 11 & 26 & $1 ?$ \\
\hline
\end{tabular}

* valor más bajo registrado.

** valor más alto registrado. 
un paciente. En la literatura aparece descrita la presencia de esferocitosis en el $65 \%$ de los casos ${ }^{3}$.

La esplenomegalia en la EH del RN no es un hecho muy relevante tal como sucedió en nues. tros enfermos.

Otro elementu clinico de gran importancia es el antecedente familiar de $\mathrm{EH}$ en uno de los padres, que en nuestra casuística est uvo presente en el $71 \%$. No siempre el antecedente de EH familiar es evidente. por lo cual es necesario buscarlo preguntando por antecedente de EH o de esplenectomia o realizando el examen hematológicu a los padres.

La fragilidad osmótica puede estar normal en la EH neonatal, si se la compara con los valores establecidos para adultos, pero es francamente aumentada si se la compara con curvas propias del RN. como lo ha demostrado Schröter ${ }^{3}$.

La expresión clínica de la EH en el período neonatal, no permite predecir una evolución grave a posteriori, como se pudiera deducir por la severidad de la anemia que presentan los $\mathrm{RN}^{18}$. Así parece acontecer también con nuestros pacientes, cinco de los cuales tienen un seguimiento suficiente y cuya evolución ha sido semejante a los casos diagnosticados después del período neonatal. El glóbulo rojo del $\mathrm{RN}$ tiene numerosas condiciones que lo hacen más susceptible a la hemolisis. Sólo mencionaremos algunos de estos factores: menor deformabilidad ${ }^{19}$, disminución del consumo de glucosa por glicolisis disminuida ${ }^{20}$, mayor sensibilidad a la injuria oxidativa y a la hemólisis osmótica 21,22 .

El diagnóstico de la EH en el RN ofrece dificultades ya que existen numerosas condicio. nes mucho más frecuentes que producen ictericia en el RN. El hemograma al demostrar la esterocitosis es el examen más valioso para el diagnó . tico diferencial de un cuadro de ictericia neonatal no explicado especialmente si hay anemia. Hay que tener en cuenta, sin embargo, que la incom. patibilidad ABO, la sepsis y algunas enzimopatias congénitas pueden evolucionar con esferocitosis de grado variable.

La incompatibilidad $\mathrm{ABO}$ es $40^{\circ}$ a 50 veces más frecuente que la EH. La prueba de Coombs habitualmente es negativa o ligeramente positiva de modo que no es de gran utilidad en el diagnóstico. Tampoco se soluciona el problema con el estudio de la fragilidad osmótica ya que puede estar alterada en la incompatibilidad ABO si se acompaña de esferocitosis importante. La confirmación diagnóstica se obtendría al comprobar la EH en uno de los padres que está presente en un alto porcentaje. La confirmación definitiva puede obtenerse ulteriormente al estudiar los enfermos en el período lactante. La esferocitosis por incompatibilidad ABO desapa. rece a los 2-3 meses lo que no sucede en la EH en que la esferocitosis persiste durante toda la vida al igual que las alteraciones de las pruebas de laboratorio (fragilidad osmótica, autohemolisis, etc.).

En todos los casos en que se ha confirmado el diagnóstico de $\mathbf{E H}$ es importante advertir a los padres la alta probabilidad de que nuevos hijos hereden el rasgo $y$ sufran hemolisis patológica en e] período neonatal que manejada adecuadamente se controla sin mayores riesgos.

\section{RESUMEN}

Se analizan las características clínicas y hematológicas de $7 \mathrm{RN}$ portadores de $\mathrm{EH}, 5$ de los cuales tenían antecedentes familiares positivos. Los 7 casos presentaron ictericia precoz con ascenso importante de la bilirrubinemia que obligó a usar fototerapia en todos y en 3 a realizar exsanguinotransfusión. Los 7 enfermos presentaron anemia importante que debić tratarse con transfusión de glóbulos rojos. La mayoría de los casos presentó como evolución una forma leve o moderada tal como sucede en la EH diagnosticada más tardíamente. Se acusan los datos de la literatura que coinciden con los hallazgos anotados. Como referencia se analizan otros 29 casos de $\mathrm{EH}$ diagnosticados en edades más tardias encontrando el antecedente de ictericia neonatal patológica en el $44,8 \%$ lo que sugiere que la hemolisis con manifestaciones clínicas está presente en el período neonatal con mucho más frecuencia de la diagnosticada. Se pone énfasis en que debe investigarse la $\mathrm{EH}$ en todos los casos de ictericia neonatal de causa no explicada y especialmente si se acompaña de anemia. Deben investigarse los antecedentes familiares de EH y hacer un estudio hematológico completo que incluya el examen de fragilidad osnótica. El diagnóstico diferencial más importante es con la enfermedad hemolítica por in. compatibilidad ABO.

\section{REFERENCIAS}

1. Weed, R.: Hercditary spherocytosis. Atch Intern Med 135: 1316,1975 .

2. Morton N.E., Mc Kinney A.A.: Genetics of spherocytosis. Am J. Hum Genet 14: 170, 1962.

3. Schröter W, Kashnit: E.: Diagnosis of hereditary spherocytosis in newborn infants. I Pediatr 103: 460,1983

4. Agarwat, M.B., Kumnd, P.M., Mehta, B.C.: Hereditary spherocytosis in a neonate. Indian Pediatr 16 : 359,1979 . 
5. Rencorer, G., Vargas L., Garcia H.: Fsferocitosis hereditaria. Bol Hosp SJ Dios 26: 293, 1979 (Chile).

6. Stamey, C.H., Diamod, L.: Congenital Hemolytic anemia in the newbron. Am J Dis Child 94:616, 1957

7. Trucco, J,L., Brown, A,K.: Neonatal manifestations of hereditary spherocytosis. Am J Dis Child I13: $263,1967$.

8. Burman, $D$.: Congenital spherocytosis in infancay Arch Dis Child 33: 335, 1958.

9. Erlandson, M, Higartner, $M$.: Hemolytic disiase in the neonatal period and early infancy. I Pediatr 54: $566,1959$.

10. Rody, $R$.: Two cases of hereditary spherocytosis manifested in the newborn period. I Pediatr 44: $213,1954$.

11. Berheim, M., Francois, R, Mouriquend, C, Germain D., Germier, $M_{\text {.: }}$ Les manifestations neonatales de la maladie de Minkowski Chauffard. Ann Pediat? (Paris) 37: 476, 1960.

12. Shapiro, C.M., Josephson, A.M. Rozengurg, S, Koufmon, A.: Hereditary Spherocytosis in the neonatal period. J Pediatr 50: 308, 1957

13. Lelong, M., Alagille, D., Dorment, $J .:$ La forme icterique neonatale de la maladie de Minkowskiet Chauffard. Arch Franc Pediatr 16: 577, 1959

14. Betke: Icterus neonatorum gravis als Manifestation einer hereditarem Spherocytose. Z Kinderheilk 78 : $359,1956$.

15. Macaulay, $D_{1}:$ Acholuric jaundice in a newborn infant. Arch Dis Child 26: 241, 1951.

16. Oisen, J.: Hereditary spherocytosis with kernicterus. Acta Paediati 49:453,1960.

17. Robinson, G.C.: Hereditary spherocytosis in infancy. J Pediatr 50: 446, 1957.

18. Lux, S. Glader, B.: Disorders of the red cell membrane en Xathan, D., Oski, F.A.; "Hematolosy of infancy and Chilhood". Philadelphia, Saunders 1981 , pág. 500.

19. Gross, G.P., Hathoway, W.E.: Fetal erythrocyte deformability. Pediat. Res. 6: 593, 1972.

20. Oski, F.A., Snith, C.A.: Apparent inappropiate glucose comsumption for cell age. Pediatres 41 : $473,1968$.

21. Schwartz, E., Gill, F.: Hematology of the newborn en Williams W., Beutler, E, Erslev, A, Luchtman, M.: Hernatology, New York, Mc Graw Hill J983, pp. 40.

22. Robinson, G., Phillips, R., Prytonsky: Spherocytosis and increased fragility occurring in exythro. blastosis fetalis associated with $\mathrm{ABO}$ incompatibility. Pediatrics 7: 164, 1951 .

23. Kostinas, J.E., Cont, W.E.F, Wetzel, R.A.: Autohemolysis of oord blood in congenital spherocytosis and ABO incompatibility. J Pediatr 70: $273,1967$. 